Damit das Leben weitergeht

\section{Olanzapin von der Akut- bis zur Langzeittherapie}

Olanzapin steht seit 15. März 2004 auch als intramuskuläre Darreichungsform für die Akuttherapie zur Verfügung. Durch die neue intramuskuläre Applikationsmöglichkeit lassen sich die Vorteile dieses modernen Neuroleptikums nun noch besser auch in der unmittelbaren Akutphase der Schizophrenie bzw. der manischen Episoden nutzen. Olanzapin i.m. ${ }^{1}$ zeichnet sich durch eine schnelle Symptomkontrolle sowie eine gute Verträglichkeit aus. Für die orale Therapie bietet Olanzapin in Form der sich rasch auflösenden Schmelztablette VeloTab ${ }^{T M}$ eine Compliance-fördernde Therapieoption, sowohl für den Akutbereich als auch - nach erfolgreicher Akuttherapie - für die Langzeittherapie.

A kut psychotische Patienten sind oft stark agitiert, feindselig und wenig krankheitseinsichtig. Häufig gefährden sie sich und ihre Mitmenschen, sodass rasches Handeln indiziert ist. In der Vergangenheit wurde in der Akuttherapie der Schizophrenie mangels Alternativen meist auf hoch potente klassische Neuroleptika zurückgegriffen. Doch diese sind mit einem hohen Risiko extrapyramidal-motorischer Nebenwirkungen behaftet. Dadurch machen viele Patienten bereits in der initialen Therapiephase traumatisierende Erfahrungen und ihre Compliance wird langfristig aufs Spiel gesetzt.

\section{Solides Fundament für den Therapieerfolg}

Olanzapin i.m. ${ }^{1}$ ist angezeigt zur schnellen Beherrschung von Agitation und gestörtem Verhalten bei Patienten mit Schizophrenie oder manischen Episoden, wenn eine orale Therapie nicht geeignet ist. Wie eine doppelblinde, randomisierte und plazebokontrollierte Multicenterstudie an 311 Patienten belegte, bewirkt Olanzapin i.m. eine zuverlässige und einschätzbare Symptomkontrolle. Gemessen wurde die Verminderung der Agitation von schizophrenen Patienten anhand der PANSS-Excited-Component. Deutlich überlegen war Olanzapin i.m. gegenüber Haloperidol i.m. aufgrund des besonders raschen Wirkeintritts. Sowohl 15 als auch 30 und

\footnotetext{
${ }^{1}$ Zyprexa ${ }^{\circledR}$ i.m., Lilly
}

45 Minuten nach der ersten Injektion war Olanzapin statistisch signifikant schneller wirksam als die Vergleichssubstanz ( $\mathrm{p}=0,01)$ (Abb. 1). Somit kommt es zur raschen Beruhigung des Patienten mit Olanzapin i.m. Über den Verlauf von 120 Minuten wird die akute Erregung durch Olanzapin ebenso effektiv und anhaltend kontrolliert wie durch Haloperidol. Sowohl unter Olanzapin (10 mg/Inj.) als auch unter Haloperidol i.m. (7,5 mg/Inj.) besserte sich die Agitation gemessen an der PANSS-Excited-Component zwei Stunden nach der Injektion in vergleichbarem Ausmaß (-7,7 vs. -7,6) (4).

Ein weiteres wichtiges Argument für das moderne Olanzapin

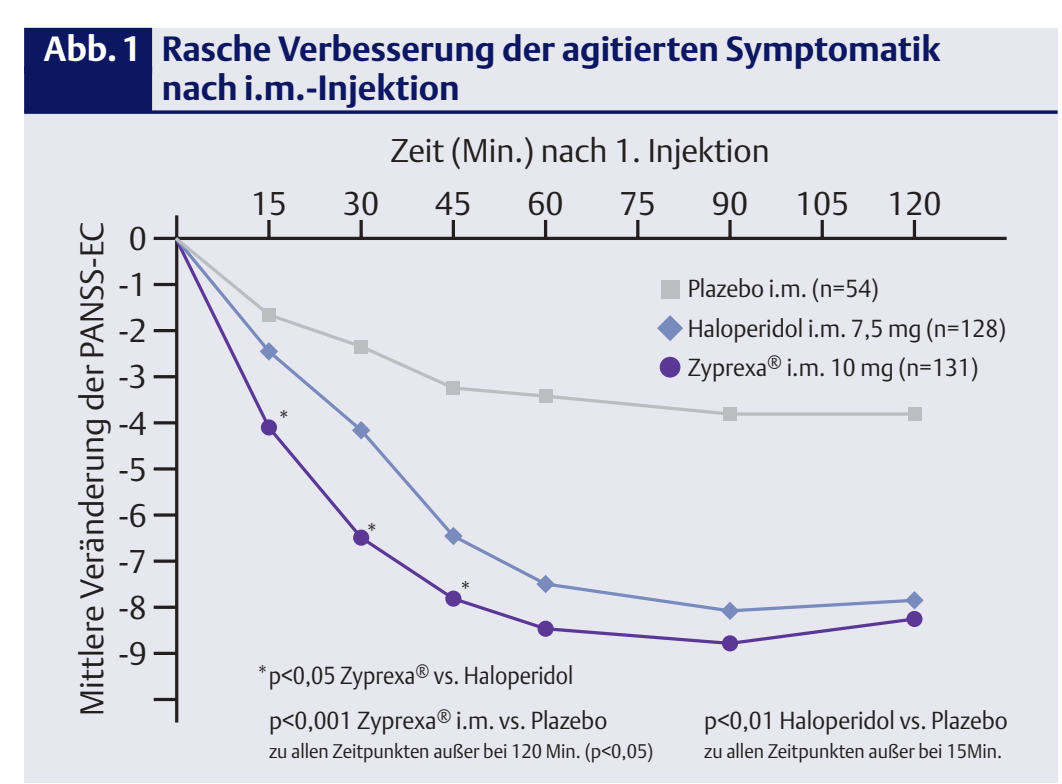

Sowohl nach 15 als auch nach 30 und 45 Minuten ist Olanzapin i.m. statistisch

signifikant wirksamer als Haloperidol i.m. (nach 4). i.m. ist die gute Verträglichkeit. Denn bei keinem der mit Olanzapin i.m. behandelten Patienten trat eine akute Dystonie auf (vs. Haloperidol $7,1 \%, p=0,001)$. Auch wurden Anticholinergika, die bei jedem fünften Haloperidol-Patienten (20,6\%) erforderlich waren, bei den mit Olanzapin i.m. behandelten Patienten genauso selten eingesetzt wie unter Plazebo (4,6\% vs. $3,7 \%)$.

Olanzapin i.m bietet in der täglichen Praxis somit ein gutes und sehr praktikables Werkzeug, um den agitierten Patienten zu beruhigen und ein „Wieder-klar-denken-Können“ zu ermöglichen. Initial wird eine Einzeldosis von $10 \mathrm{mg}$ Olanzapin i.m. empfohlen. Aufgrund der klaren Dosis-Wirkbeziehung kann die Dosis an den klinischen Zustand angepasst werden (1). Die tägliche Höchstdosis beträgt $20 \mathrm{mg}$, wobei innerhalb von 24 Stunden nicht mehr als drei Injektionen vorgenommen werden sollten (2).

\section{Problemloser Übergang auf orale Erhaltungstherapie}

Sind die Patienten nach erfolgreicher Hochakuttherapie kooperativer, bietet sich die Umstellung auf orales Olanzapin an. Mit der sich im Mund oder in Flüssigkeit rasch auflösenden VeloTab ${ }^{\mathrm{TM}}$ steht eine Olanzapin-Tablette zur Verfügung, die sowohl für die Akut- als auch für die 
Langzeittherapie geeignet ist. Die VeloTab $^{\mathrm{TM}}$ ist praktisch in der Verwendung und muss von Anfang an nur einmal täglich genommen werden. Das orale Olanzapin gewährt eine konstant fortdauernde zuverlässige und einschätzbare Symptomkontrolle (Abb. 2), ohne erhöhtes Risiko von schweren Nebenwirkungen in Form von akuten Dystonien. In der direkten Vergleichsstudie berichteten die Patienten nach Umstellung auf orales Olanzapin anders als unter Haloperidol - weder über akute Dystonien noch Akathisien (3).

\section{Langfristiger Therapieerfolg}

Dank der zuverlässigen und einschätzbaren Symptomkontrolle und der guten Verträglichkeit von Olanzapin können den Patienten bereits initial traumatische Erfahrungen erspart werden. Damit ist es möglich, den Grundstein für einen langfristig positiven Therapieverlauf bereits in der Akuttherapie zu legen.

Ulla Satzger-Harsch, Stuttgart

\section{Abb. 2 Harmonischer Übergang in die orale Olanzapin-Therapie}

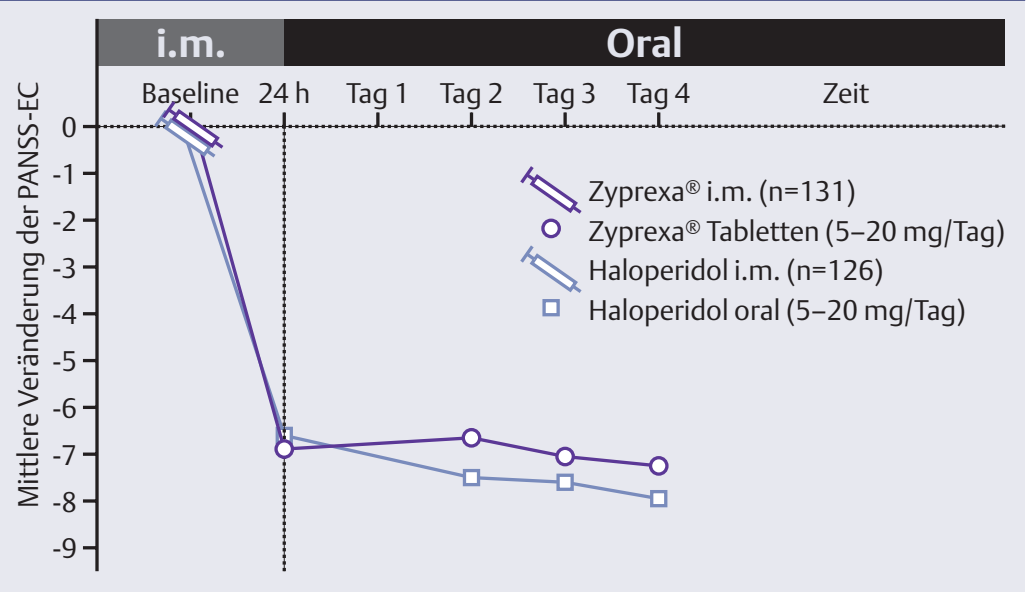

Anhaltend zuverlässige und einschätzbare Symptomkontrolle nach der Umstellung von Olanzapin i.m. auf orales Olanzapin (nach 3 ).

\section{Literatur}

1. Breier et al. A Double-Blind, PlaceboControlled Dose-Response Comparison of Intramuscular Olanzapine and Haloperidol in the Treatment of Acute Agitation in Schizophrenia. Arch Gen Psychiatry 2002; 59 : 441-448

2. Fachinformation Zyprexa ${ }^{\circledR} 10 \mathrm{mg}$ Pulver

3. Wright et al. A Comparison of the Effi- cacy and Safety of Olanzapine Versus Haloperidol During Transition from Intramuscular to Oral Therapy. Clinical Therapeutics 2003; 25 (5): 1420-1428

4. Wright et al. Double-Blind, PlaceboControlled Comparison of Intramuscular Olanzapine and Intramuscular Haloperidol in the Treatment of Acute Agitation in Schizophrenia, Am J Psychiatry 2001; 158 (7): 1149-1151
$\mathrm{M}$ ein Lebenslauf ist untrennbar mit den Maltesern verbunden. Ich verdanke den Maltesern so viel, das möchte ich jetzt zurückgeben". Mit diesem Bekenntnis zu den Maltesern nahm jetzt Prof. Dr. med. Peter Knuth die Berufung zum ehrenamtlichen ärztlichen Leiter Rettungsdienst für den Bezirk Köln der Malteser an, die ihm vom Vorsitzenden der Geschäftsführung der Malteser Hilfsdienst gGmbH, Karl Prinz zu Löwenstein, ausgesprochen wurde. Seit über 40 Jahren ist Prof. Knuth auch ehrenamtlich für die Helfer unter dem achtspitzigen Kreuz aktiv. 1964 gründete der gebürtige Bamberger, den es früh in das Rheinland gezogen hatte, die Malteser-Gliederung in Linnich. Zwischen 1975 und 1997 flog er mehr als 1800 Einsätze auf dem kölner Rettungshubschrauber „Christoph 3“. Zunächst als Rettungssanitäter, um sich sein Medizinstudium zu finanzieren, später als Notarzt. Lange Zeit war Prof. Knuth auch Leitender Notarzt des weltweiten Malteser-Rückholdienstes (Köln)

\section{„Mit den Maltesern groß geworden“ \\ Prof. Dr. med. Peter Knuth zum ärzt- lichen Leiter Rettungsdienst berufen}

und gab wesentliche Impulse zur Qualitätssicherung im notfallmedizinischen Bereich. Für dieses langjährige Engagement wurde Prof. Knuth von Bezirksgeschäftsführer Hans-Gerd Achnitz bei dieser Gelegenheit auch mit der Jubiläumsmedaille des Großmeisters des Souveränen Malteserritterordens ausgezeichnet.

Prof. Knuth, der in Wiesbaden und Berlin als ärztlicher Geschäftsführer des Bundesverbandes der Internisten arbeitet und in Düsseldorf einen Lehrauftrag im Fachbereich Medizin ausübt, engagiert sich seit Jahren als Mitglied in den wesentlichen Gremien der Intensiv- und Notfallmedizin in Deutschland. Das bundesweit zertifizierte Qualitätsmanagementsystem im Rettungsdienst fachlich $\mathrm{zu}$ begleiten und weiterzuentwickeln ist das vorrangige Ziel. „Qualität rettet Leben“, da ist sich Prof. Knuth ganz sicher. N

Quelle: Presse-Information der Malteser im Bezirk Köln, Februar 2004.

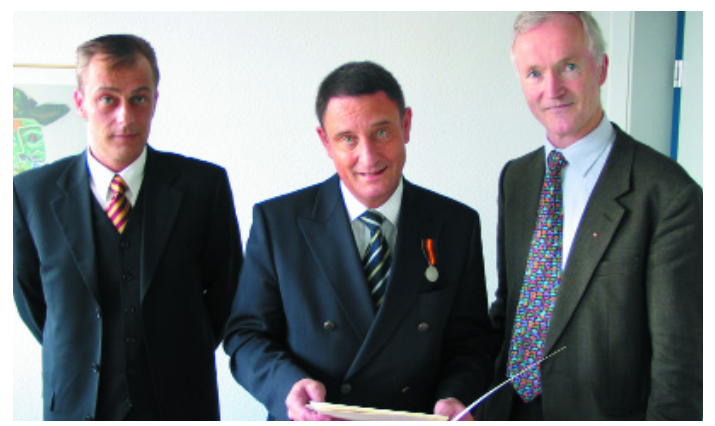

Prof. Dr. med. Peter Knuth (m.) wurde von Karl Prinz zu Löwenstein ( $r$.) zum neuen ärztlichen Leiter Rettungsdienst im Bezirk Köln berufen und von Hans-Gerd Achnitz (I.) mit der Jubiläumsmedaille des Malteserritterordens ausgezeichnet. 